\title{
UM ALGORITMO DE NEGOCIAÇÃO AUTOMATIZADO BASEADO EM UMA ANÁLISE GRÁFICA, PODE APRESENTAR UM BOM RESULTADO?
}

\section{CAN AN AUTOMATED TRADING ALGORITHM BASED ON A GRAPHICAL ANALYSIS PRESENT A GOOD RESULT?}

\section{Recebimento: 13/06/2019 - Aceite: 10/01/2020 - Publicação: 28/02/2020 Processo de Avaliação: Double Blind Review}

\author{
Alexandre Martins Carvalho ${ }^{1}$ \\ Graduado em Administração pela Universidade Federal de Uberlândia (UFU) \\ carvalhoalexandre@outlook.com.br
}

Flávio Barboza

Universidade Federal de Uberlândia (UFU)

flmbarboza@ufu.br

\section{José Augusto Fiorucci}

Doutor em Estatística pela Universidade Federal de São Carlos (UFSC)

Professor da Universidade de Brasília (UnB)

jafioruci@gmail.com

\section{RESUMO}

Investimentos baseados na análise técnica vêm sendo utilizados com maior frequência para examinar o desempenho estratégico das negociações automatizadas por meio de um robô (algoritmo) de investimentos, em particular, usando os indicadores Parabolic SAR e Fibonacci. Este trabalho utilizou a avaliação de cenários para posteriormente comparar seus resultados em relação à estratégia de buy and hold. Os cenários se diferenciam em relação à utilização de fatores de risco, timeframes e níveis de preço. Backtests foram realizados por um período compreendido entre Janeiro de 20015 e Abril de 2017 para comparar as estratégias. Como resultado, foi percebido que a utilização da análise técnica por meio da negociação automatizada pode resultar em lucros superiores à buy and hold. Entretanto, tal forma de negociação apresenta um alto nível de volatilidade.

Palavras-chave: Negociação Automatizada; Análise Técnica; Fibonacci; Parabolic SAR.

\section{ABSTRACT}

Investments based on technical analysis have been used more frequently to examine the strategic performance of automated negotiations through an investment algorithm, in

\footnotetext{
${ }^{1}$ Autor para correspondência: Universidade Federal de Uberlândia (UFU): Av. João Naves de Ávila, 2121 Bloco 1F, Campus Santa Mônica, Uberlândia-MG CEP 38400-902 - Brasil.
}

Revista ENIAC Pesquisa, Guarulhos (SP), V.9, n.1, fev.- jul. 2020. 
particular using parabolic SAR indicators and Fibonacci. This work used the scenario evaluation to later compare their results in relation to the buy and hold strategy. Scenarios differ from the use of risk factors, timeframes and price levels. Backrests were carried out for a period from January 20015 to April 2017 to compare the strategies. As a result, it was noticed that the use of technical analysis through automated trading can result in profits higher than buy and hold. However, such a form of trading presents a high level of volatility.

Keywords: Automated Trading. Technical Analysis. Fibonacci. Parabolic SAR.

\section{INTRODUÇÃO}

De acordo com Fama (1965) há correntes de pensamentos sobre finanças que divergem no modo de pensar o comportamento dos preços futuros: por um lado têm-se que o preço futuro é aleatório, e, por outro, que preços seguem certos padrões.

O primeiro grupo compõe pensadores que acreditam na hipótese do mercado eficiente. Nessa corrente, Lo (2007) ressalta duas figuras principais: Eugene Fama e Paul Samuelson. Segundo Fama (1970), um mercado no qual os preços refletem a totalidade das informações disponíveis é chamado de eficiente, podendo-se segregar na forma forte, semi-forte e fraca. Para Bruni e Fama (1998), os preços sempre refletem as informações disponíveis, pois o mercado é composto por investidores racionais, inexistindo, portanto, oportunidades de ganhos anormais.

Por outro lado, temos autores que afirmam que é possível obter ganhos anormais a partir de análises que encontrem imperfeições de mercado. Ohlson (1990), Frankel e Lee (1998), e Piotroski (2000), encontraram evidências que é possível utilizar análises contábeis para prever preços futuros dos ativos. Já Abarbanell (1991) constatou que as previsões dos analistas não contemplam todas informações de mercado, contradizendo a premissa de que investidores são racionais na tomada de decisão.

Brown, Goetzmann e Kumar (1998) afirmam que Charles Dow deu inícios aos estudos em análise técnica (AT), ao observar padrões em uma carteira de ativos. A partir dos seus estudos, seu sucessor, William Hamilton, terminou de escrever a Teoria de Dow.

Levich e Thomas III (1993), apresentaram evidências de que métodos para trade com base na AT podem gerar retornos positivos. Neely, Weller e Dittmar (1997) utilizaram técnicas de programação para operar estratégias de investimentos com base na AT, obtendo como resultado retornos positivos acima do normal.

Dentre os vários indicadores técnicos existentes, destacam-se o Fibonacci e Parabolic

Revista ENIAC Pesquisa, Guarulhos (SP), V.9, n.1, fev.- jul. 2020. 
SAR. De acordo com Roberts (1959), o estudo da AT pode se pautar em evidências de estudos empíricos, bem como processos naturais observáveis. Para Gaucan (2011), a sequência numérica Fibonacci, presente tanto na matemática quanto na natureza, cria padrões (razão de ouro), os quais são usados para formar o indicador utilizado na AT. De acordo com Bhattacharya e Kumar (2006), as razões calculadas pelo método Fibonacci indicam possíveis pontos de mudança da direção da tendência.

O indicador Parabolic SAR (Parabolic Stop and Reverse, PSAR), tem seu uso, de acordo com Nan e Sun (2011), com o objetivo de determinar tendência no preço do ativo estudado. Ainda segundo estes autores, é indicado que utilize o PSAR para definir o instante de uma operação de compra (venda), caso aponte tendência de alta (baixa).

Este trabalho tem como objetivo entender como os indicadores técnicos Fibonacci e Parabolic Sar funcionam em conjunto, para em seguida montar uma estratégia de operação automatizada, testando sua eficácia.

Para Sherstov e Stone (2006), o estudo que envolve a negociação automatizada tem uma extrema importância prática, devido a mudança das operações no mercado financeiro decorrente a partir do desenvolvimento tecnológico. Adicionalmente, tais avanços possibilitam desenvolver métodos automatizados para realizar suas aplicações, servindo como alternativa à ação humana.

Os indicadores técnicos, portanto, servem de base para o desenvolvimento de uma estratégia criada em forma de algoritmo para ser operada de forma independente da ação humana. Sendo assim, o algoritmo funciona como uma espécie de robô de investimento, operando a partir de uma estratégia pré-determinada sem a necessidade de interferências direta de pessoas.

\section{REVISÃO DA LITERATURA}

A bibliografia consulta neste trabalho levou em conta teorias sobre o comportamento dos preços de mercado. Dentre elas, as Hipótese do Mercado Eficiente (HME), discordando da possibilidade de prever movimentos futuros nos preços, enquanto do outro lado encontram-se as análises fundamentalistas e técnicas, desenvolvendo estudos que identifica padrões que possibilitam a predição dos movimentos futuros.

Revista ENIAC Pesquisa, Guarulhos (SP), V.9, n.1, fev.- jul. 2020. 


\subsection{HIPÓTESE DO MERCADO EFICIENTE}

Fama (1965) diz que uma pergunta controversa permeia os estudos no campo das finanças, servindo como base em estudos com resultados opostos: até que ponto preços passados de ativos podem influenciar seus preços futuros? De um lado têm-se os grafistas mostrando que é possível encontrar padrões para prever preços, outros autores se baseiam na random walk theory, na qual diz que o comportamento de preços futuros é independente de preços passados.

A origem da HME deu-se início com estudos na década de 1960 com Eugene Fama e Paul Samuelson (LO, 2007), sendo "Proof that Properly Antecipated Prices Fluctuate Randomly" (SAMUELSON, 1965), o pioneiro. Nele, o autor constata que o preço futuro não segue nenhuma correlação com os preços passados.

De acordo com Lo (2007), a HME assume que um mercado em que as informações são distribuídas de forma eficiente, evitando a possibilidade de se operar com informações privilegiadas, não seria possível prever as alterações de preço de um determinado ativo se as mesmas fossem corretamente incorporadas nos preços.

A primeira regra do mercado é que as firmas alocam seus recursos em produção ou investimentos e os investidores alocam seus recursos em ativos das firmas, assumindo que o valor de seus ativos está inteiramente refletido a partir de toda informação que há no mercado, formando assim um mercado eficiente (FAMA, 1970). Bruni e Famá (1998) argumentam preços sempre irão refletir todas informações relevantes, uma vez que os mercados são compostos por investidores racionais, inexistindo oportunidades de ganhos anormais.

Em termos gerais, Fama (1970) propõe três formas para identificar a hipótese do mercado eficiente: fraca, semi-forte e forte. De acordo com o autor, a HME em sua forma fraca analisa apenas o preço histórico, não representando uma boa forma de análise de uma empresa para realizar investimento. Já a forma semiforte é verificada quando os preços são ajustados pelas informações públicas das empresas, enquanto a forma forte considera que existem grupos com monopólios de acesso a informações que afetam o preço do ativo.

A HME foi de grande importância para consolidação e evolução dos estudos em finanças, entretanto há correntes de pensamentos dentro da Teoria de Finanças que contrapõe seus argumentos. Existem vários estudos que apresentam evidências contrárias a HME, as quais o autor classifica de anomalias dos mercados de capitais, dentre elas: as análises técnicas (ou

Revista ENIAC Pesquisa, Guarulhos (SP), V.9, n.1, fev.- jul. 2020. 
gráficas) e fundamentalistas - a qual utiliza como base informações contábeis, financeiras, setoriais e econômicas (BRUNI; FAMÁ,1998).

Seguindo os preceitos das análises fundamentalistas, o estudo de informações contábeis é relevante ao definir o valor de um ativo, havendo uma relação entre variáveis contábeis com o preço do ativo (OHLSON, 1990). Outros autores, como Frankel e Lee (1998) encontraram evidências de que informações contábeis está correlacionada com o preço dos ativos.

Piotroski (2000), estudou um método de análise de ações baseado e seus indicadores contábeis, criando uma estratégia com base em uma análise fundamental para formar seu portfólio. A estratégia de investimento utilizada, que define seu portfólio de acordo com suas análises fundamentalistas, obteve um retorno de $23 \%$ a.a.

Com o objetivo de averiguar as projeções dos analistas, Abarbanell (1991) percebeu que, mesmo que os analistas utilizem apenas as informações, negligenciando a mudança de preços, a probabilidade de suas projeções ser menor (ou maior) que o realizado aumenta caso haja um crescimento (diminuição) no preço. Como resultado, o autor conferiu que as projeções dos analistas não refletem por inteiro todas as informações.

\subsection{A TEORIA DE DOW}

A Teoria de Dow teve início com as publicações do editor do The Wall Street Journal, Chales Dow. Esta teoria tem como premissa que o funcionamento do mercado acionário segue de acordo com padrões que persistem entre tendências. Mesmo que as análises possam ser dificultadas por oscilações de curto prazo, o desenho da curva de preços passados permite ao analista identificar qual será a tendência do mercado (BROWN; GOETZMANN; KUMAR, 1998).

Outro aspecto importante levantado por Brown, Goetzmann e Kumar (1998) é que tais tendências são ocasionadas em função da irracionalidade do investidor, o qual não incorpora tal efeito em suas expectativas para o preço do ativo. Logo, os movimentos do mercado seguem padrões em até três instâncias de tendência, tendo a primeira como reflexo dos fundamentos da atividade econômica daquele mercado, bem como as alterações de preços futuros.

Revista ENIAC Pesquisa, Guarulhos (SP), V.9, n.1, fev.- jul. 2020. 


\subsection{ANÁLISE TÉCNICA}

A análise de preços históricos de ativos é uma forma que muitos analistas tentam encontrar padrões gráficos para projetar o que irá acontecer com os preços futuros. A teoria de Dow seria uma das várias evidências da importância da análise técnica (AT) (ROBERTS, 1959).

Os indicadores gráficos gerados pela AT para criar projeções ou identificar tendências utilizam apenas o comportamento passado dos preços dos ativos. Osler (2003) defende que a AT é uma boa ferramenta para o curto prazo, porém não como instrumento para definir no longo prazo. Isso porque existe uma tendência dos investidores em definir ordens em números redondos, gerando um agrupamento de ordens em um determinado ponto, permitindo aos indicadores de AT identificar tais padrões gráficos.

Neely, Weller e Dittmar (1997) utilizaram técnicas de programação para operar estratégias de trading com base na AT, obtendo como resultado retornos positivos acima do normal. Levich e Thomas III (1993) também evidenciaram resultados similares.

A chave da AT, para Bhattacharya e Kumar (2006) é exatamente identificar, a partir de padrões no gráfico do fluxo de preço, o melhor ponto para comprar ou vender uma ação com base na probabilidade de um mesmo evento passado acontecer com os preços desse ativo novamente.

\subsection{RAZÕES DE FIBONACCI}

De acordo com Roberts (1959), a AT pode se pautar em evidências, bem como processos observáveis na natureza. Uma ferramenta de AT que se baseia em evidências encontradas na natureza é o estudo do indicador Fibonacci.

A sequência numérica de Fibonacci advém de padrões observáveis tanto na matemática como na natureza. Assim, investidores acreditam que o mercado financeiro apresenta padrões com a estrutura coerente com as razões de ouro encontradas a partir da sequência de Fibonacci (GAUCAN, 2011).

O indicador de Fibonacci assume que os preços tendem a se comportar formando ciclos e padrões, bem como explicado por Bhattacharya e Kumar (2006), ao se formar uma tendência, a sua força diminui em um certo ponto, tornando-se estável e reverte para depois voltar ao seu

Revista ENIAC Pesquisa, Guarulhos (SP), V.9, n.1, fev.- jul. 2020. 
movimento inicial. Além disso, os níveis calculados pelo indicador detectam possíveis valores que sugerem a mudança na direção da tendência, indicando potenciais preços de compra ou venda.

\subsection{PARABOLIC SAR}

O indicador Parabolic SAR (PSAR) tem sua aplicabilidade, de acordo com Nan e Sun (2011), com o objetivo de determinar tendência no preço do ativo estudado. Seu valor segue uma fórmula que considera fatores de aceleração em relação a preços anteriores e pontos extremos. Segundo Lee et al. (2010) a fórmula do PSAR é dada por:

$$
\mathrm{SAR}_{\mathrm{t}}=\mathrm{SAR}_{\mathrm{t}-1}+\mathrm{AF}\left(\mathrm{XP}-\mathrm{SAR}_{\mathrm{t}-1}\right)
$$

onde $\mathrm{SAR}_{\mathrm{t}}$ é o valor do indicador PSAR no tempo $\mathrm{t}, \mathrm{AF}=$ fator de aceleração, $\mathrm{XP}=$ ponto extremo. Dessa maneira, PSAR é igual ao preço máximo operado em tendências de alta ou o

menor preço operado em tendências de baixa. Quanto maior o ponto extremo, maior a probabilidade de haver uma reversão de tendência, sendo que a cada novo pico encontrado, o AF é incrementado. Lee et al. (2010) utiliza o AF sendo 0,02 até o ponto máximo de 0,2 (XP).

\subsection{NEGOCIAÇÃO AUTOMATIZADA: ROBÔ DE INVESTIMENTOS}

A natureza eletrônica das transações do mercado possibilita ao investidor desenvolver métodos automatizados para realizar suas aplicações, servindo como alternativa à ação humana (SHERSTOV; STONE, 2006).

Neely, Weller e Dittmar (1997) utilizaram algoritmos para testar a AT, obtendo como resultado retornos positivos acima do normal. Além deles, Teixeira e Oliveira (2010) também desenvolveram uma metodologia de operação automatizada por meio de AT, gerando retornos superiores à estratégia de buy and hold.

Revista ENIAC Pesquisa, Guarulhos (SP), V.9, n.1, fev.- jul. 2020. 


\section{METODOLOGIA}

A pesquisa teve como proposta avaliar o desempenho de um algoritmo na realização de operações no mercado financeiro. Para tanto, o robô foi testado na plataforma MetaTrader4.

O algoritmo baseia-se em dois indicadores técnicos: o Parabolic SAR (PSAR) e o indicador de Fibonacci. O primeiro basicamente é utilizado como um gatilho para toda estratégia, identificando qual é a possível tendência futura, enquanto o segundo define o ponto de execução das operações.

Inicialmente, o robô passa a analisar o movimento dos preços pelo PSAR, para verificar a tendência do mercado. Como exemplo, observa-se na figura 1 o funcionamento do indicador, no qual o ponto A mostra o momento em que o PSAR (pontos em torno dos candles) indica mercado em queda, pois há a inversão - os pontos que estavam por baixo sofrem a reversão e então aparecem por cima dos candles - enquanto o ponto B mostra um possível início de tendência de alta, ou seja, o gráfico mostra os preços históricos da taxa de câmbio Dólar/Real no mercado FOREX, destacando os pontos de inversão da tendência identificados pelo PSAR.

Figura 1 - Gráfico FOREX, PSAR.

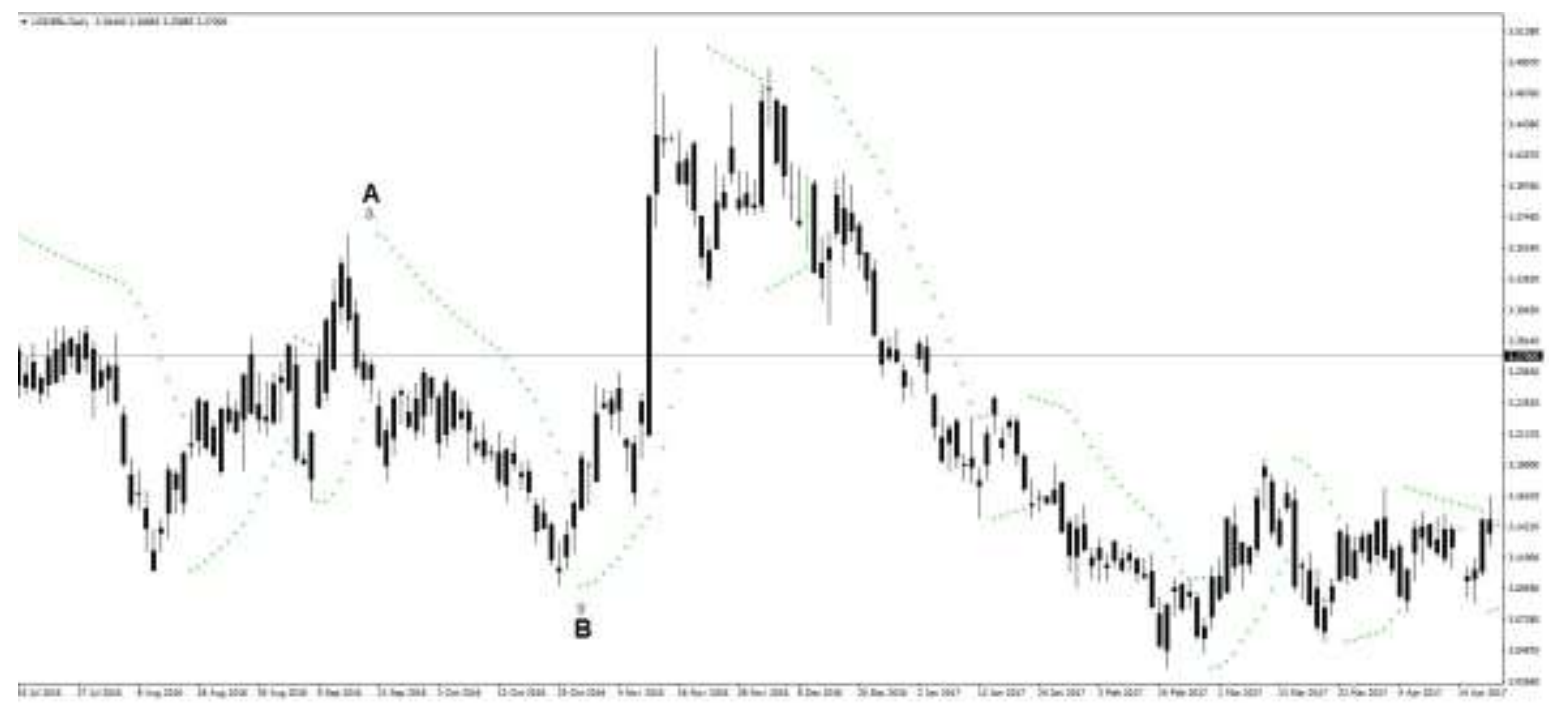

Fonte: Elaborado pelos autores.

Como um dos requisitos para o funcionamento do robô, foi necessário definir alguns parâmetros que têm o papel de definir sistematicamente o seu funcionamento, tais como o padrão de tempo do gráfico (timeframes), que indica de intervalo de tempo o gráfico gera um 
novo ponto de análise, pontos de entrada e saída da operação, fornecidos pelo indicador de Fibonacci.

Sendo assim, quando PSAR evidenciar um início de tendência na evolução a estratégia poderia começar a operar com compra ou venda. Para operar aproveitando a tendência de preços, em acordo com o estudo de Nan e Sun (2011), as ordens foram realizadas de acordo com o sinal dado pelo PSAR.

Uma vez indicada a tendência, o robô calcula os níveis de preço sugeridos pelo indicador de Fibonacci. Assim, verifica 50 períodos anteriores ao alinhamento do PSAR, em busca do maior e menor valores negociados, sendo estes os níveis de $0 \%$ e $100 \%$ do indicador.

O movimento de preços baseados em Fibonacci pressupõe, basicamente, que ao final de uma tendência de alta, o movimento se retrai e sugere que existem pontos que promovem a inversão, voltando a tendência inicial (BHATTACHARYA; KUMAR, 2006). Baseando-se nos pontos definidos pelas razões Fibonacci $(23,6 \%, 38,3 \%, 50 \%, 61,8 \%, 76,4 \%$ 85,4\% e 100\%) o robô foi programado para operar em função de fatores de riscos pré-determinados em cada uma das razões apresentadas.

Como gestão de riscos, a partir do momento que os pontos A e B são conhecidos, instalase um stop-loss (SL) 10\% abaixo (acima) do ponto A, caso a operação inicial for de compra (venda).

A figura 2 mostra o Fibonacci plotado, incluindo o SL, ou seja, a cotação do ativo USDBRL (taxa de câmbio Dólar/Real negociada no mercado FOREX) mostrando em detalhes a formação dos níveis calculados pelo indicador Fibonacci. Assim, só são executadas as ordens a partir da definição dos pontos A e B, operando após a correção prevista para então voltar a tendência inicial. Tal correção ocorreria do ponto B até A2, retornando para a tendência inicial (A2 a B2), como mostra a figura 3, que ilustração de uma movimentação do preço da taxa de câmbio Dólar/Real no mercado FOREX, incluia um exemplo de análise técnica feita pelo robô (cenários de tendência A-B, B-A2 e A2-B2, e os níveis dados pelas razões de Fibonacci).

Após o lançamento de ordem, a conclusão da operação acontece quando o preço do ativo atinge o nível de $161,8 \%$ do movimento de expansão de Fibonacci ou quando o preço do ativo retrai abaixo do nível acima de onde a ordem foi lançada. Ou seja, se a ordem foi lançada no nível $85,4 \%$ e o preço após, ultrapassar o nível de $100 \%$, retrai abaixo do mesmo, há a realização da posição.

Revista ENIAC Pesquisa, Guarulhos (SP), V.9, n.1, fev.- jul. 2020. 
Figura 2 - USDBRL (taxa de câmbio Dólar/Real negociada no mercado FOREX)

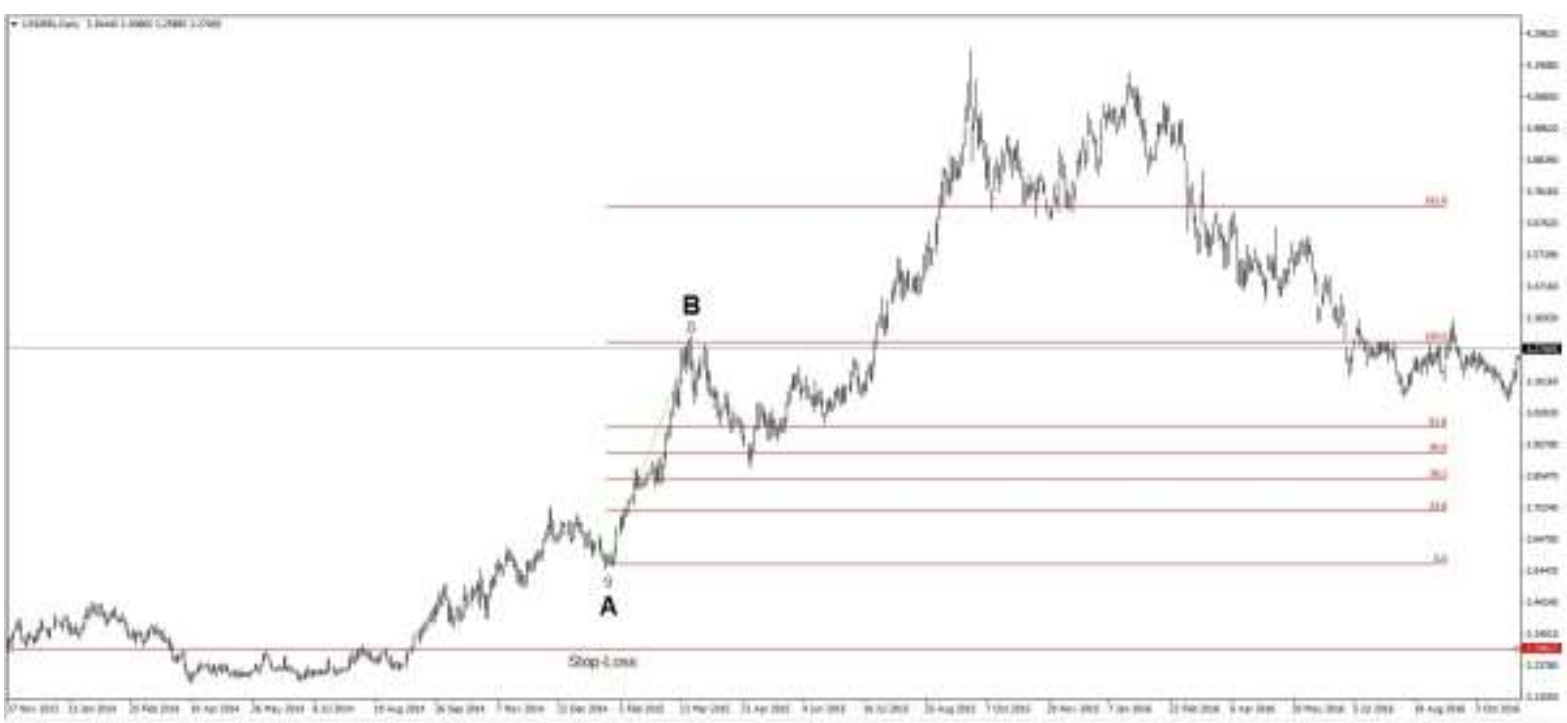

Fonte: Elaborado pelos autores.

Figura 3 - Cenários de tendência A-B, B-A2 e A2-B2, (Fibonacci).

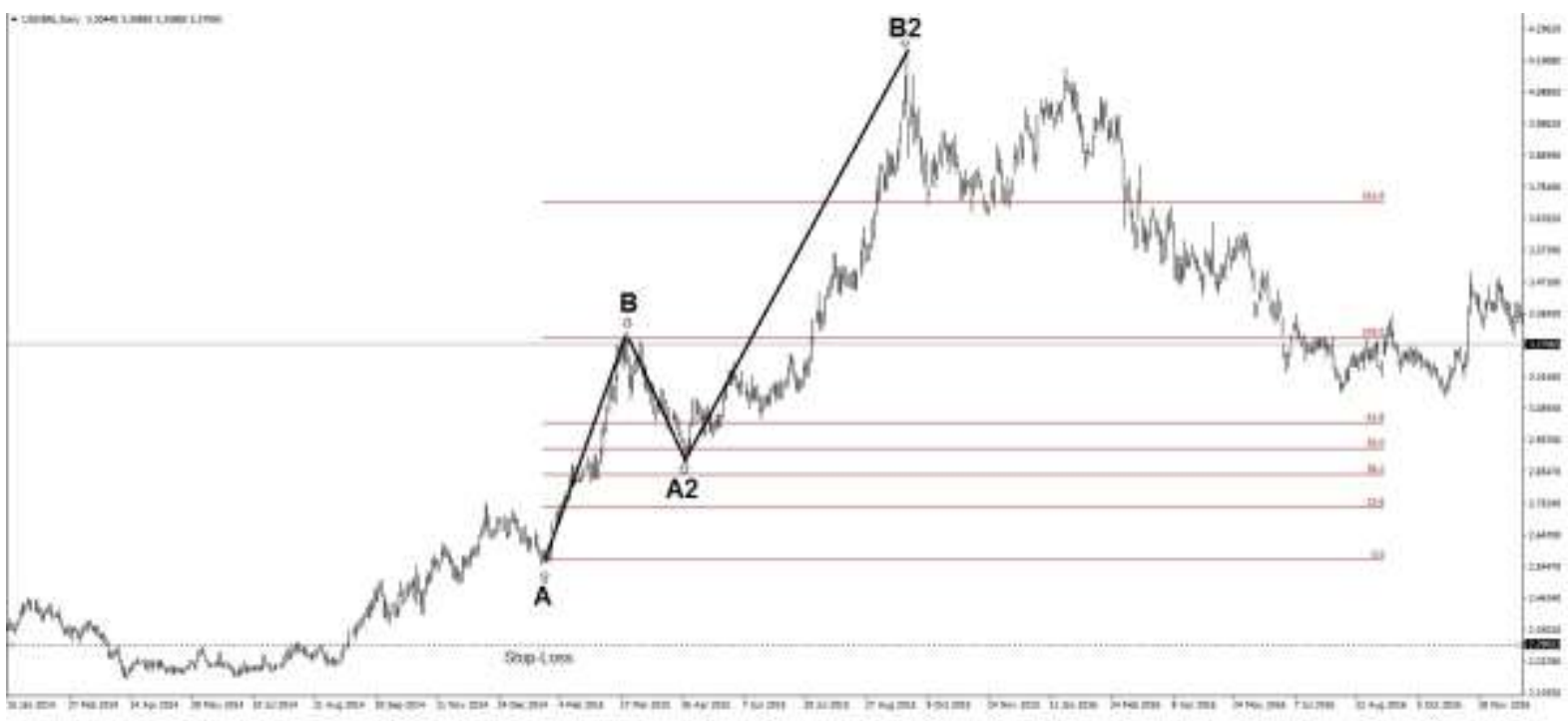

Fonte: Elaborado pelos autores.

Como base para a gestão de risco do robô, é necessário definir qual fator de risco para

Revista ENIAC Pesquisa, Guarulhos (SP), V.9, n.1, fev.- jul. 2020. 
cada uma das razões Fibonacci antes de rodar o backtest. O cálculo de quanto foi operado é feito com base em quanto de risco foi pré-determinado para cada um dos níveis e o saldo em conta na hora da operação.

Os testes foram realizados simulando operação no mercado de câmbio FOREX, operando por meio do ativo dólar em relação ao real (USDBRL). O período análise foi de 02 de janeiro de 2015 até 28 de abril de 2017.

Para definição dos cenários analisados, o estudo baseou-se em uma sistemática para alocação de riscos para cada razão Fibonacci. Primeiramente, foi analisado a estratégia alocando risco individualmente para cada razão Fibonacci, em cada uma das timeframes possíveis, com o intuito de identificar em quais cenários haveriam um resultado positivo.

Para isso, foi realizado um teste para cada nível Fibonacci com 0,01 de fator de risco. Dessa forma, foi separado quais razões Fibonacci e quais timeframes geraram mais resultados positivos e quais geram resultados negativos. Como mostra a tabela 1, o gráfico diário apresentou resultados positivos de desempenho para simulações em cada nível de risco adotadas de acordo com o padrão indicado pelas razões de Fibonacci.

Tabela 1 - Timeframes.

\begin{tabular}{ccccc}
\hline Razões de & & \multicolumn{2}{c}{ Timeframe } & \\
Fibonacci & 1 dia & 4 horas & 1 hora & 30 min \\
\hline $100 \%$ & Lucro & Prejuízo & Prejuízo & Prejuízo \\
$85,40 \%$ & Lucro & Prejuízo & Prejuízo & Prejuízo \\
$76,40 \%$ & Lucro & Prejuízo & Prejuízo & Prejuízo \\
$61,80 \%$ & Lucro & Prejuízo & Prejuízo & Prejuízo \\
$50 \%$ & Prejuízo & Prejuízo & Prejuízo & Prejuízo \\
$38,30 \%$ & Lucro & Prejuízo & Prejuízo & Prejuízo \\
$23,60 \%$ & Prejuízo & Prejuízo & Prejuízo & Prejuízo \\
\hline
\end{tabular}

Fonte: Elaborado pelos autores.

Sendo assim, foi possível definir uma estratégia que alocasse risco apenas onde foi encontrado resultados positivos. Para entender como o fator de risco altera a eficiência da operação, outro cenário foi estudado a partir das razões que geraram resultados positivos, porém com um fator de risco elevado. Além desses cenários, foi realizado um teste alocando risco para as razões Fibonacci que resultaram em retornos negativos, os Fatores de Risco estipulados em cada nível da razão de Fibonacci nos cenários de simulação que foram testados. Como exemplo, um fator de 0,05 significa que a operação aloca 5\% do capital disponível na ordem enviada com 
o preço limitado ao percentual [dado pela razão de Fibonacci] do preço do ativo considerando a máxima e mínima (100\% e 0\%) dentro dos últimos 50 períodos anteriores a inversão apontada pelo indicador PSAR. (Tabela 2)

Tabela 2 - Fatores de Risco PSAR.

\begin{tabular}{cccccc}
\hline $\begin{array}{c}\text { Razões de } \\
\text { Fibonacci }\end{array}$ & $\mathrm{A}$ & $\mathrm{B}$ & $\mathrm{C}$ & $\mathrm{D}$ & $\mathrm{E}$ \\
\hline $\mathbf{1 0 0 \%}$ & 0,05 & 0,10 & 0,05 & 0,00 & 0,03 \\
$\mathbf{8 5 , 4 0 \%}$ & 0,05 & 0,10 & 0,04 & 0,00 & 0,02 \\
$\mathbf{7 6 , 4 0 \%}$ & 0,05 & 0,10 & 0,03 & 0,00 & 0,00 \\
$\mathbf{6 1 , 8 0 \%}$ & 0,05 & 0,10 & 0,02 & 0,00 & 0,00 \\
$\mathbf{5 0 \%}$ & 0,00 & 0,00 & 0,00 & 0,10 & 0,00 \\
$\mathbf{3 8 , 3 0 \%}$ & 0,05 & 0,10 & 0,01 & 0,00 & 0,01 \\
$\mathbf{2 3 , 6 0 \%}$ & 0,00 & 0,00 & 0,00 & 0,10 & 0,00 \\
\hline
\end{tabular}

Fonte: Elaborado pelos autores.

Em suma, foram simulados 5 cenários, descritos na tabela 2, para analisar o funcionamento indicadores técnicos em conjunto operando a taxa de câmbio USD/BRL a partir de um capital inicial de $\$ 10.000,00$, com diferentes fatores de risco em cada uma das razões Fibonacci. Os dados históricos utilizados foram em relação ao período de 02/01/2015 até 28/04/2017, adotando apenas o timeframe de 1 dia.

\section{ANÁLISE DOS RESULTADOS}

\subsection{ANÁLISE DE DESEMPENHO DO BACKTEST CONSIDERANDO O CENÁRIO}

A

A tabela 3 mostra um resumo do teste realizado com o robô de uma simulação realizada com o uso da estratégia automatizada pelo robô, tendo o cenário A como referência para a gestão de risco.

Revista ENIAC Pesquisa, Guarulhos (SP), V.9, n.1, fev.- jul. 2020. 
Tabela 3 - Referência para a gestão de risco.

\begin{tabular}{lc}
\hline Indicadores de Desempenho & Resultado \\
\hline Rentabilidade & $55.95 \%$ \\
\hline Posição Final & $\$ 15,594.53$ \\
Máxima Perda & $\$ 5,142.57$ \\
Total de Operações & 190 \\
Média de Operações com Lucro & $\$ 250.29$ \\
Média de Operações com Prejuízo & $\$ 249.24$ \\
Operação com Maior Lucro & $\$ 1,185.39$ \\
Operação com Maior Perda & $\$ 932.36$ \\
Operações Vendidas (ganhadoras \%) & $112(60,71 \%)$ \\
Operações Compradas (ganhadoras \%) & $78(48,72 \%)$ \\
Operações Lucrativas (\%total) & $106(55,79 \%)$ \\
\hline
\end{tabular}

Fonte: Elaborado pelos autores.

A estratégia operada para o primeiro cenário buscou um perfil mais conservador, com baixo nível de risco. Apesar do nível de risco não ser elevado, tal cenário realizou um lucro considerável $(\$ 5.594,53)$, uma rentabilidade de $55,95 \%$, porém com uma alta volatilidade, evidenciada pela maior perda consecutiva $(\$ 5.142,57)$ e pela figura 4 , representando a curva da posição de saldo disponível em relação a cada operação realizada. O Gráfico ilustrando a Evolução do Saldo Disponível (linha azul) do Cenário A. A linha verde (Equity) mostra o saldo no referido instante de tempo, considerando posições abertas, ou seja, operações ainda não encerradas. Size representa o volume de posições em aberto. O eixo horizontal descreve o número de operações realizadas.

Figura 4 - Gráfico Evolução do Saldo Disponível

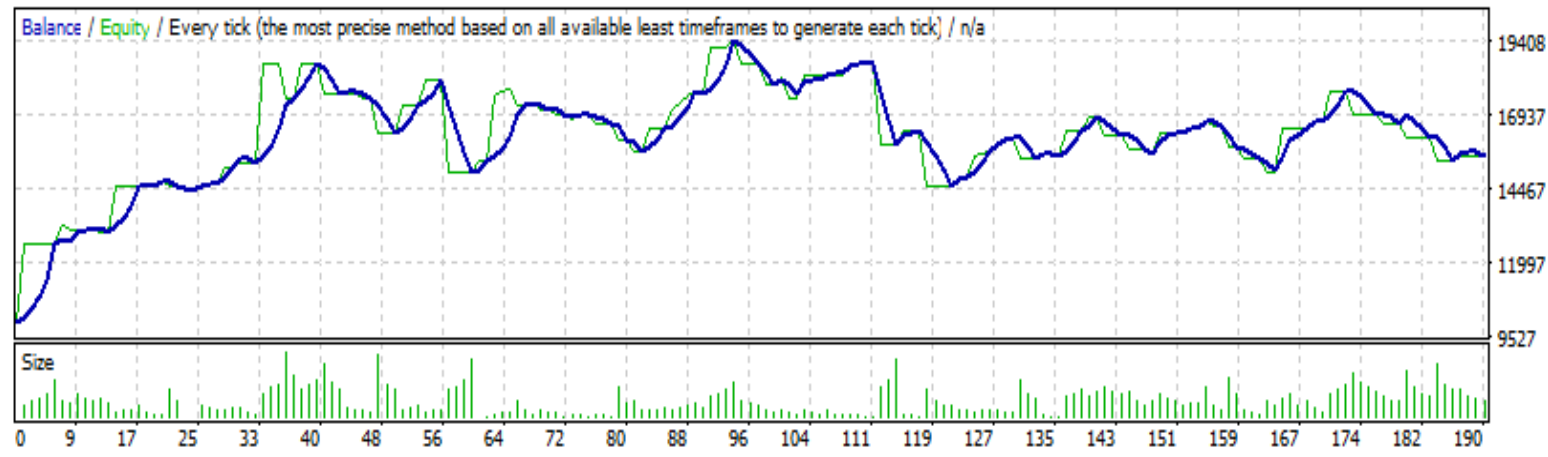

Fonte: Elaborado pelos autores.

Revista ENIAC Pesquisa, Guarulhos (SP), V.9, n.1, fev.- jul. 2020. 
Nessa estratégia foram realizadas 190 operações, sendo que 112 foram posicionamentos vendidos e 78 posições compradas, sendo que $60,71 \%$ e $48,72 \%$, respectivamente, geraram lucros. A média de resultado das operações com lucro $(\$ 250,29)$ superou a média dos retornos das operações com prejuízo $(\$ 249,39)$ em módulo por uma diferença pequena. Do total de 190 operações, $106(55,79 \%)$ tiveram resultados positivos.

\subsection{ANÁLISE DE DESEMPENHO DO BACKTEST CONSIDERANDO O CENÁRIO \\ B}

O Quadro resumo da simulação realizada com o uso da estratégia automatizada pelo robô, tendo o cenário B como referência para a gestão de risco, pode ser observado na Tabela 4.

Tabela 4 - Quadro resumo da simulação

\begin{tabular}{lc}
\hline Indicadores de Desempenho & Resultado \\
\hline Rentabilidade & $90.86 \%$ \\
\hline Posição Final & $\$ 19,086.03$ \\
Máxima Perda & $\$ 17,785.11$ \\
Total de Operações & 190 \\
Média de Operações com Lucro & $\$ 693.25$ \\
Média de Operações com Prejuízo & $\$ 766.65$ \\
Operação com Maior Lucro & $\$ 2,841.72$ \\
Operação com Maior Perda & $\$ 3,033.97$ \\
Operações Vendidas (ganhadoras\%) & $112(60,71 \%)$ \\
Operações Compradas (ganhadoras\%) & $78(48,72 \%)$ \\
Operações Lucrativas (\%total) & $106(55,79 \%)$ \\
\hline
\end{tabular}

Fonte: Elaborado pelos autores.

Para testar o efeito de um aumento do fator de risco no funcionamento do robô, a estratégia adotada no cenário 2 contou operações realizadas nas mesmas razões Fibonacci, porém com fator de risco multiplicado por 2 . Ou seja, $10 \%$ de risco nos níveis $100 \%, 85,4 \%$, $76,4 \%, 61,8 \%$, e $38,3 \%$.

Como resultado, observou-se o mesmo número de operações, vendidas e compradas, e mesmas porcentagens de acerto. Uma vez que se trata de um cenário mais arriscado, houve um resultado com rentabilidade superior, $90,86 \%$. 
A média de operações com resultado lucrativo $(\$ 693,25)$ veio abaixo (em valores absolutos) da média de operações com prejuízo $(\$ 766,65)$. A operação com maior perda $(\$ 3.033,97)$ também foi acima em comparação com operação de maior lucro $(\$ 2.841,72)$.

A posição final do segundo cenário foi de $\$ 19.086,03$. Entretanto, tal estratégia apresentou uma volatilidade alta, representada pela perda máxima de $\$ 17.785,11$. A figura 5 representa o saldo disponível à cada operação realizada, evidenciando ainda mais a volatilidade de tal estratégia, uma vez que o saldo chegou próximo aos $\$ 32.000,00$, tendo apenas $\$ 19.086,03$ como posição final.

O Gráfico ilustra a Evolução do Saldo Disponível (linha azul) do Cenário B. A linha verde (Equity) mostra o saldo no referido instante de tempo, considerando posições abertas, ou seja, operações ainda não encerradas. Size representa o volume de posições em aberto. O eixo horizontal descreve o número de operações realizadas.

Figura 5 - Gráfico Evolução do Saldo Disponível - Cenário B.

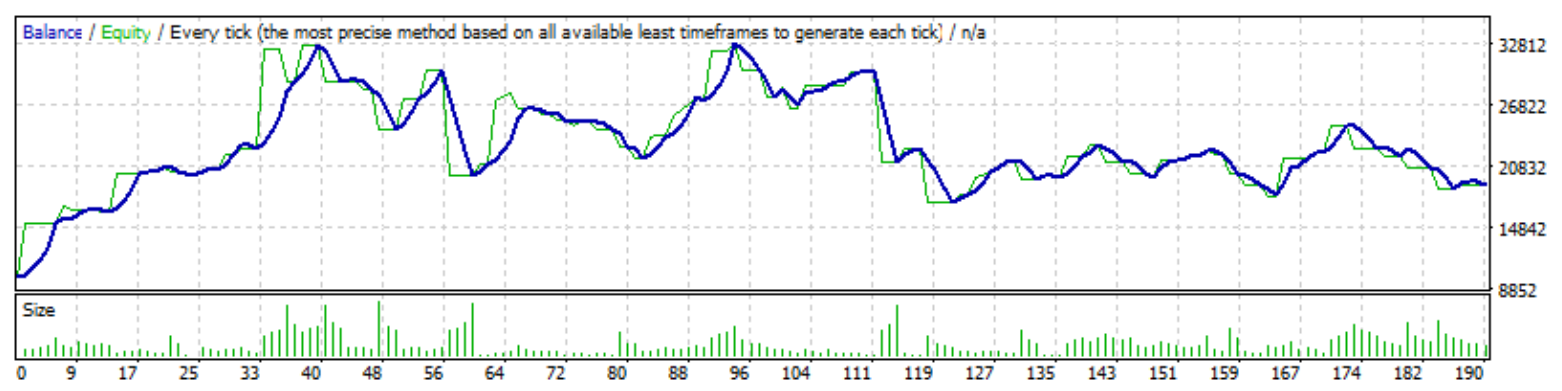

Fonte: Elaborado pelos autores.

\subsection{ANÁLISE DE DESEMPENHO DO BACKTEST CONSIDERANDO O CENÁRIO C}

Com o intuito de analisar o efeito de uma alocação de risco progressiva em relação à retração para os níveis Fibonacci, o cenário $\mathrm{C}$ apresentou diferentes fatores de riscos, porém mesmas razões que nos cenários $\mathrm{A}$ e $\mathrm{B}$, como mostra a tabela 5. Nesse cenário, a rentabilidade bruta foi de $38,97 \%$, menor que as encontradas no primeiro e segundo cenário.

Revista ENIAC Pesquisa, Guarulhos (SP), V.9, n.1, fev.- jul. 2020. 
Tabela 5-Quadro resumo estratégia automatizada pelo robô

\begin{tabular}{lc}
\hline Indicadores de Desempenho & Resultado \\
\hline Rentabilidade & $38.97 \%$ \\
\hline Posição Final & $\$ 13,897.27$ \\
Máxima Perda & $\$ 1,989.87$ \\
Total de Operações & 190 \\
Média de Operações com Lucro & $\$ 125.86$ \\
Média de Operações com Prejuízo & $\$ 112.43$ \\
Operação com Maior Lucro & $\$ 267.46$ \\
Operação com Maior Perda & $\$ 478.48$ \\
Operações Vendidas (ganhadoras\%) & $112(60,71 \%)$ \\
Operações Compradas (ganhadoras\%) & $78(48,72 \%)$ \\
Operações Lucrativas (\%total) & $106(55,79 \%)$ \\
\hline
\end{tabular}

Fonte: Elaborado pelos autores.

Como mostra a figura 6 , a terceira estratégia apresentou um nível de volatilidade baixa, com o saldo disponível seguindo uma clara tendência de alta ao longo das operações, também evidenciado pela máxima perda de apenas $\$ 1.989,88$ para os mesmos níveis de acertos e operações, vendidas e compradas, apresentadas na estratégia A e B. O Gráfico ilustra a Evolução do Saldo Disponível (linha azul) do Cenário C. A linha verde (Equity) mostra o saldo no referido instante de tempo, considerando posições abertas, ou seja, operações ainda não encerradas. Size representa o volume de posições em aberto. O eixo horizontal descreve o número de operações realizadas

Figura 6 - Gráfico Evolução do Saldo Disponível - Cenário C.

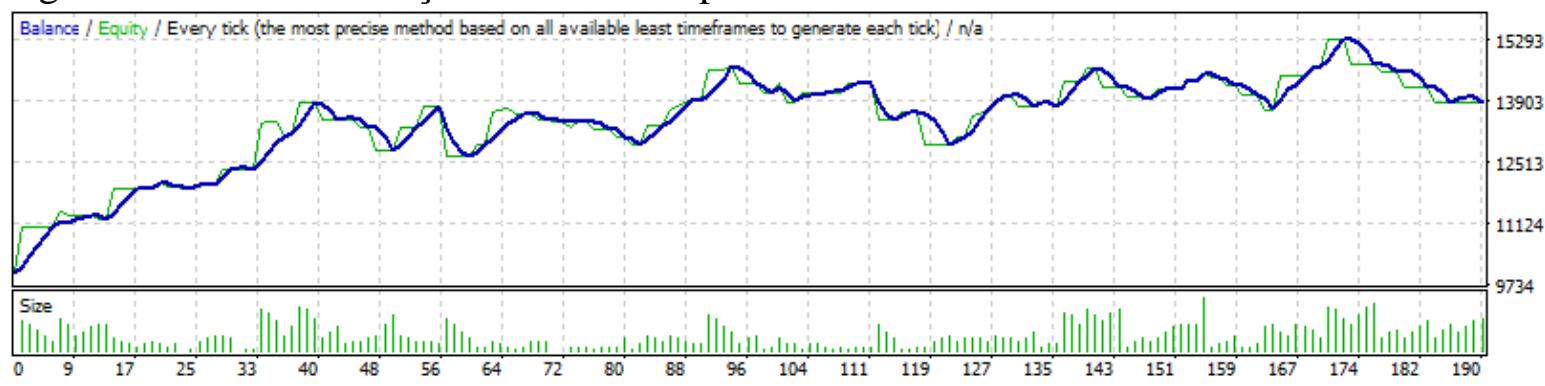

Fonte: Elaborado pelos autores.

Revista ENIAC Pesquisa, Guarulhos (SP), V.9, n.1, fev.- jul. 2020. 


\subsection{ANÁLISE DE DESEMPENHO DO BACKTEST CONSIDERANDO O CENÁRIO}

D

O quarto cenário teve como objetivo testar uma estratégia com fatores de risco nas razões Fibonacci não contempladas nos cenários anteriores. Sendo assim, foi alocado 0,1 de fator de risco nos níveis $50 \%$ e 23,6\%. A tabela 6 mostra o relatório de desempenho para este caso. A tabela 6 apresenta o resumo da simulação realizada com o uso da estratégia automatizada pelo robô, tendo o cenário D como referência para a gestão de risco.

Tabela 6 -Resumo da simulação realizada - Cenário D.

\begin{tabular}{lc}
\hline Indicadores de Desempenho & Resultado \\
\hline Rentabilidade & $-12.14 \%$ \\
\hline Posição Final & $\$ 8,786.10$ \\
Máxima Perda & $\$ 5,837.90$ \\
Total de Operações & 17 \\
Média de Operações com Lucro & $\$ 561.57$ \\
Média de Operações com Prejuízo & $\$ 634.05$ \\
Operação com Maior Lucro & $\$ 1,694.92$ \\
Operação com Maior Perda & $\$ 1,281.11$ \\
Operações Vendidas (ganhadoras\%) & $9(55,56 \%)$ \\
Operações Compradas (ganhadoras\%) & $8(37,50 \%)$ \\
Operações Lucrativas (\%total) & $8(47,06 \%)$ \\
\hline
\end{tabular}

Fonte: Elaborado pelos autores.

Como resultado, obteve-se apenas 17 operações, 8 compradas e 9 vendidas. Desse total, apenas $8(47,06 \%)$ resultou em lucro. Desse modo, resultado bruto da quarta estratégia, foi um prejuízo de $12,14 \%(\$-1.213,900)$.

\subsection{ANÁLISE DE DESEMPENHO DO BACKTEST CONSIDERANDO O CENÁRIO \\ $\mathbf{E}$}

Por último as análises operando USDBRL, o quinto cenário buscou uma estratégia com fatores de riscos mais elevados e progressivos, operando apenas nas razões $100 \%, 85,4 \%$ e 38,3\%. A performance do robô neste cenário é vista na tabela 7 apresenta o resumo da simulação realizada com o uso da estratégia automatizada pelo robô, tendo o cenário E como 
referência para a gestão de risco.

Tabela 7 - Quadro resumo - Cenário E.

\begin{tabular}{lc}
\hline Indicadores de Desempenho & Resultado \\
\hline Rentabilidade & $320.40 \%$ \\
\hline Posição Final & $\$ 42,039.51$ \\
Máxima Perda & $\$ 25,909.64$ \\
\hline Total de Operações & 113 \\
\hline Média de Operações com Lucro & $\$ 1,643.74$ \\
Média de Operações com Prejuízo & $\$ 1,916.10$ \\
Operação com Maior Lucro & $\$ 4,073.67$ \\
Operação com Maior Perda & $\$ 7,252.13$ \\
Operações Vendidas (ganhadoras\%) & $71(63,38 \%)$ \\
Operações Compradas (ganhadoras\%) & $42(59,52 \%)$ \\
Operações Lucrativas (\%total) & $70(61,95 \%)$ \\
\hline
\end{tabular}

Fonte: Elaborado pelos autores.

Constatou-se, nesta simulação, a maior rentabilidade 320,40\%, bem como o maior nível de uma perda máxima de $\$ 25.909,64$. Entretanto, mesmo com níveis elevado de perdas, é possível perceber uma tendência de alta nos níveis de saldo disponível, evidenciado pela figura 7.

Figura 7 - Evolução do Saldo Disponível do Cenário 5.

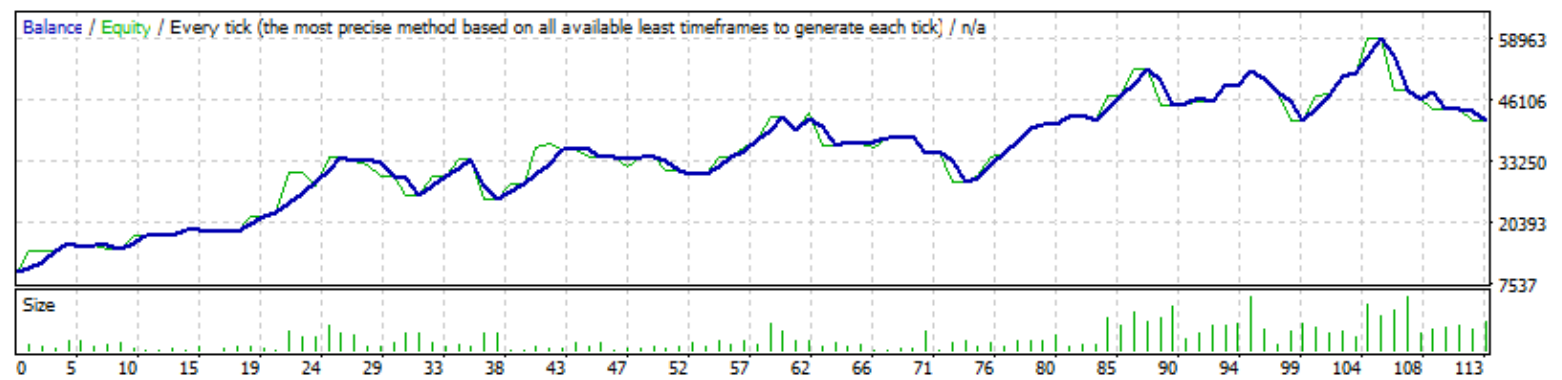

Fonte: Elaborado pelos autores.

Foram operadas 113 vezes, sendo que $70(61,95 \%)$ resultaram em lucro. Foram 71 operações vendidas e 42 operações compradas, com 63,38\% e 58,52\%, respectivamente, de assertividade. A média das operações lucrativas $(\$ 1.643,74)$ foram, em módulo abaixo da média das operações com prejuízo $(\$ 1.916,10)$.

Revista ENIAC Pesquisa, Guarulhos (SP), V.9, n.1, fev.- jul. 2020. 


\subsection{BUY AND HOLD CONTRA NEGOCIAÇÃO AUTOMATIZADA}

A estratégia buy and hold consiste na compra do ativo e sua manutenção até um período pré-determinado. Sendo assim, foi analisada a variação da cotação do dólar considerando o intervalo de tempo em que o robô operou, ou seja, de 02/01/2015 até 28/04/2017.

De acordo com dados do Banco Central (BACEN, 2017), a cotação do dólar no dia 02/01/2015 era de $\mathrm{R} \$ 2,6951$, terminando em 28/04/2017 com a cotação de $\mathrm{R} \$ 3,1761$, o que representa uma variação positiva de $17,85 \%$.

Observa-se, portanto, que apenas a estratégia utilizada no cenário 4 obteve um resultado aquém da estratégia de buy and hold. Sendo assim, a utilização de negociação automatizada pode ser uma alternativa de investimento como parte do portfólio do investidor, tendo em vista seus retornos, volatilidade e riscos.

\section{CONCLUSÕES}

De acordo com a "Hipótese do Mercado Eficiente", as informações disponíveis são racionalmente precificadas no preço dos ativos de forma que impossibilite um ganho anormal por meio de operações no mercado de ações. Entretanto, há correntes de pensamentos que formam análise fundamentalista e gráfica que afirma que é possível prever movimentos de preços futuros com base em indicadores pré-existentes, indicando uma irracionalidade no mercado. Sabendo disso, o estudo objetivou analisar um método de negociação automatizada com base em indicadores técnicos.

Uma mesma estratégia que aloque riscos em determinados níveis Fibonacci pode resultar em resultados não-lineares se operada com diferentes fatores de riscos. Sendo assim, é essencial que além da simulação de diversos cenários é necessária uma gestão de risco eficiente, definindo stop-loss e take-profits que vá de acordo com o perfil de risco de cada investidor.

Além disso, observa-se que há uma elevada volatilidade nos resultados das operações, tornando um investimento arriscado. Tendo isso em vista, o investidor que optar por operar com base em indicadores técnicos deve dedicar apenas parte de seu portfolio, para que sua liquidez e saúde financeira não seja totalmente comprometida.

Sendo assim, é possível chegar a conclusão de que a análise técnica utilizada como base para criação de estratégias operadas por robôs de investimentos pode resultar em lucro. 
Entretanto, é necessário realizar diversos backtests de cada estratégia, simulando diversos cenários antes de operar em definitivo.

\section{REFERÊNCIAS}

ABARBANELL, J. S. Do analyst's earnings forecasts incoporate information in prior stock price changes?. Journal of Accounting and Economics, v.14, n. 2, p. 147-165, 1991.

BACEN - Banco Central do Brasil. Disponível em: < https://www.bcb.gov.br/>. Acesso em $11 / 11 / 2017$.

BHATTACHARYA, S.; KUMAR, K.. A computational exploration of the efficacy of Fibonacci Sequences in technical analysis and trading. Annals of Economics and Finance, v. 7, n. 1, p. 185, 2006. Disponível em: < https://research.bond.edu.au/en/publications/acomputational-exploration-of-the-efficacy-of-fibonacci-sequence>. Acesso em 12/10/2019.

BROWN, S. J.; GOETZMANN, W. N.; KUMAR, A. The Dow theory: William Peter Hamilton's track record reconsidered. The Journal of Finance, v. 53, n. 4, p. 1311-1333, 1998. Disponível em: <https://miami.pure.elsevier.com/en/publications/the-dow-theory-williampeter-hamiltons-track-record-reconsidered $>$. Acesso em 12/10/2019.

BRUNI, A. L.; FAMÁ, R. Eficiência, previsibilidade dos preços e anomalias em mercados de capitais: teoria e evidências. Caderno de Pesquisas em Administração, v. 1, n. 7, p. 71-85, 1998. Disponível em: < https://www.revistas.usp.br/rege/article/download/36638/39359/>. Acesso em 12/10/2019.

FAMA, E. F. The behavior of stock-market prices. The Journal of Business, v.38, n.1, p. 34105, 1965. Disponível em: < https://www.jstor.org/stable/2350752>. Acesso em 12/10/2019.

FAMA, E. F. Efficient capital markets: A review of theory and empirical work. The Journal of Finance, v. 25, n. 2, p. 383-417, 1970. Disponível em: <https://www.jstor.org/stable/2325486>. Acesso em 12/10/2019.

FRANKEL, R.; LEE, C. M. Accounting valuation, Market expectation and cross-sectional stock returns. Journal of Accounting and Economics, v.25, n.3, p. 283-319, 1998. Disponível em:

<https://econpapers.repec.org/article/eeejaecon/v_3a25_3ay_3a1998_3ai_3a3_3ap_3a283319.htm>. Acesso em 12/10/2019.

GAUCAN, V. How to use Fibonacci retracement to predict forex market. Journal of Knowledge Management, Economics and Information Technology, v.1, n. 2, 2011. Disponível em: < https://ideas.repec.org/a/spp/jkmeit/1134.html >. Acesso em 12/10/2019.

LEE, S. J.; AHN, J. J., OH, K. J.; KIM, T. Y. Using rough set to support investment strategies of real-time trading in futures market. Applied Intelligence, v. 32, n. 3, p. 364-377, 2010. Disponível em: < https://yonsei.pure.elsevier.com/en/publications/using-rough-set-to-support- 
investment-strategies-of-real-time-tra>. Acesso em 12/10/2019.

LEVICH, R. M.; THOMAS III, L. R. The significance of technical trading-rule profits in the foreign exchange market: a bootstrap approach. Journal of international Money and Finance, v. 12, n. 5, p. 451-474, 1993. Disponível em: <https://econpapers.repec.org/article/eeejimfin/v_3a12_3ay_3a1993_3ai_3a5_3ap_3a451-

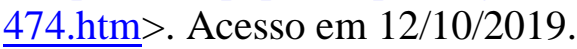

LO, A. W. Efficient markets hypothesis. In: BLUME, L.; DURLAUF, S. The New Palgrave: A Dictionary of Economics, ed. 2. New York: Palgrave McMillan, 2007. Disponível em: <http://web.mit.edu/Alo/www/Papers/EMH_Final.pdf >. Acesso em 12/10/2019.

NAN, X.; SUN, X. Automatic stock market forecasting system based on extended language template model. Journal of Information \& Computational Science, v. 8, n. 1, p. 112-118, 2011.

NEELY, C. J.; WELLER, P. A. Technical trading rules in the European monetary system. Journal of International Money and Finance, v. 18, n. 3, p. 429-458, 1999.

NEELY, C.; WELLER, P.; DITTMAR, R. Is technical analysis in the foreign exchange market profitable? A genetic programming approach. Journal of Financial and Quantitative Analysis, v. $32, \quad$ n. 04 , p. 405-426, 1997. Disponível em: <https://www.cambridge.org/core/journals/journal-of-financial-and-quantitativeanalysis/article/is-technical-analysis-in-the-foreign-exchange-market-profitable-a-geneticprogramming-approach/D959AD60856CECB9DB136AEFF6AD48FF>. Acesso em $12 / 10 / 2019$.

OHLSON, James A. A synthesis of security valuation theory and the role of dividends, cash flows, and earnings. Contemporary accounting research, v. 6, n. 2, p. 648-676, 1990. Disponível em: 〈https://doi.org/10.1111/j.1911-3846.1990.tb00780.x $>$. Acesso em 12/10/2019.

OSLER, C. L. Currency orders and Exchange rate dynamics: an explanation for the predictive success of technical analysis. The Journal of Finance, v. 58, n. 5, p.1791-1820, 2003. Disponível $<$ http://people.brandeis.edu/ cosler/documents/Currency\%20Orders $\% 20$ and $\% 20$ Exchange $\% 2$ 0Rate\%20Dynamics.pdf $>$. Acesso em 12/10/2019.

PIOTROSKI, Joseph D. Value investing: The use of historical financial statement information to separate winners from losers. Journal of Accounting Research, v. 38. pp. 1-41, 2000. Disponível em: <https://pdfs.semanticscholar.org/9dc9/b030da18ab28d1e1a1bbf7e33fb5911f189e.pdf $>$. Acesso em 12/10/2019.

ROBERTS, Harry V. Stock- Market "Patterns" And Financial Analysis: Methodological Suggestions. The Journal of Finance, v. 14, n. 1, p. 1-10, 1959. Disponível em: <https://doi.org/10.1111/j.1540-6261.1959.tb00481.x >. Acesso em 12/10/2019. 
SAMUELSON, Paul A. Proof That Properly Anticipated Prices Fluctuate Randomly. Industrial Management Review, v. 6, p. 41-49, 1965. Disponível em: <http://jrigb.jj.cqut.edu.cn/_local/6/35/CE/516B5F529EC5AAF4B9C1FFD5C4B_A532FC8 A_B39E2.pdf>. Acesso em 12/10/2019.

SHERSTOV, A. A.; STONE, P. Three automated stock-trading agents: A comparative study. In: International Workshop on Agent-Mediated Electronic Commerce. Springer Berlin Heidelberg, 2004. $\quad$ p. 173-187. <https://link.springer.com/chapter/10.1007/11575726_13 > . Acesso em 12/10/2019.

TEIXEIRA, L. A.; OLIVEIRA, A. L. I. A method for automatic stock trading combining technical analysis and nearest neighbor classification. Expert Systems With Applications, v. 37, n. $10, \quad$ p. 6885-6890, 2010. Disponível em: <https://dl.acm.org/doi/10.1016/j.eswa.2010.03.033 >. Acesso em 12/10/2019. 